\title{
70. A Method of Solution of Linear Partial Differential Equations of the Second Order with Function Coefficients
}

\author{
By Moritsune UchiYama \\ Department of Mathematics, Yokohama Municipal University, Yokohama \\ (Comm. by Z. Suetuna, M.J.A., July 13, 1959)
}

Although the linear partial differential equations of the second order with constant coefficients of the terms of the second order have already been solved $[1,6]$, it seems to have not yet done to solve the linear partial differential equations of the second order with function coefficients of the terms of the second order. In this note, a method will be proposed for reducing the latter problem to the former succeeding in solving the linear partial differential equations of the second order with function coefficients:

$$
g^{i j}(x) \frac{\partial^{2} u}{\partial x^{i} \partial x^{j}}+b^{l}(x) \frac{\partial u}{\partial x^{l}}+c(x) u=f(x)
$$

under a certain condition for the function coefficients. The basic principle is to transform the parallel coordinates $\left(x^{l}\right)$ to the II-geodesic parallel coordinates $\left(\xi^{l}\right)$ of Prof. T. Takasu $[2-4]$ and to render (0.1) to the form:

$$
\varepsilon_{l} \frac{\partial^{2} u}{\partial \xi^{l} \partial \xi^{l}}+\bar{b}^{l}(\xi) \frac{\partial u}{\partial \xi^{l}}+\bar{c}(\xi) u=\bar{f}(\xi),\left(\varepsilon_{l}= \pm 1\right) .
$$

I. Recapitulation of extended affine geometry of Prof. T. Takasu

1. II-Geodesic curves in an affine space $A^{n}$. Let $\left(x^{l}\right),(l=1,2$, $\cdots, n)$ be parallel coordinates of a point in an affine space $A^{n}$. Set

$$
\omega^{l} \stackrel{\text { def }}{=} \omega_{m}^{l}(x) d x^{m}, \quad(l, m, \cdots, p, \cdots=1,2, \cdots, n),
$$

where the Pfaffians are assumed to be not exact (an-holonomic) in general. Throughout this paper, we assume that the affine space $A^{n}$ is a differentiable manifold of class $C^{\nu}(\nu=$ positive integer or $\nu=\infty$ or $\nu=\omega)$ and that $\omega^{l}$ are linearly independent:

$$
\left|\omega_{m}^{l}(x)\right| \neq 0 \text { in } A^{n} .
$$

The assumption (1.2) will be justified by the actual existence of extended affine transformation in Art. 2.

N.B. We can apply our theory also to the case of the atlas of differentiable manifolds in the sense of S. S. Chern [7] and C. Ehresmann [8].

For the given $\omega_{m}^{l}(x)$, we introduce $\Omega_{l}^{q}(x)$ by the conditions

$$
\Omega_{l}^{p} \omega_{q}^{l}=\delta_{q}^{p}, \quad \Omega_{q}^{l} \omega_{l}^{p}=\delta_{q}^{p},
$$

and set 


$$
\Lambda_{h k}^{p} \stackrel{\text { def }}{=} \Omega_{l}^{p} \frac{\partial \omega_{h}^{l}}{\partial x^{k}}=-\omega_{h}^{l} \frac{\partial \Omega_{l}^{p}}{\partial x^{k}} .
$$

We are considering not only the affine space $A^{n}(x)$, but also the differentiable manifold $\left\{\omega_{m}^{l}(x)\right\}$, thus the sum-manifold:

$$
A^{n}(x)+\left\{\omega_{m}^{l}(x)\right\} \text {. }
$$

Take a curve $x^{p}=x^{p}(t)$, ( $t=$ affine length), which we shall study.

We can readily prove the identity:

$$
\frac{d}{d t} \frac{\omega^{l}}{d t}=\omega_{q}^{l}\left(\frac{d^{2} x^{q}}{d t^{2}}+\Lambda_{h k}^{q} \frac{d x^{h}}{d t} \frac{d x^{k}}{d t}\right) .
$$

Thus we have

$$
\frac{d}{d t} \frac{\omega^{2}}{d t}=0 \rightleftarrows \frac{d^{2} x^{r}}{d t^{2}}+A_{h k}^{r} \frac{d x^{h}}{d t} \frac{d x^{k}}{d t}=0 .
$$

What (1.7) gives, we will call the II-geodesic curves (read: 'geodesic curves of the second kind') corresponding to $\omega_{m}^{l}(x)$.

The first differential equation (1.7) is integrated readily:

$$
\begin{gathered}
\omega^{l}=a^{l} d t, \quad\left(a^{l}=\text { const. }\right), \\
\xi^{l} \stackrel{\text { def }}{=} \int \frac{\omega^{l}}{d t} d t=a^{l} t+c^{l}, \quad\left(c^{l}=\text { const. }\right),
\end{gathered}
$$

which exists in $A^{n}$, which is of class $C^{\nu}$.

From (1.9), we see that the curves represented by (1.7) or (1.9) behave as for meet and join like straight lines.

Now we may write:

$$
d \xi^{l}=\omega^{l}=a_{m}^{l}(x) d x^{m}, \quad\left(a_{m}^{l}(x) \stackrel{\text { def }}{=} \omega_{m}^{l}(x)\right),
$$

notwithstanding. $\omega^{l}$ is not holonomic (exact) in general.

The first equation of (1.7) may be rewritten:

$$
\frac{d^{2} \xi^{l}}{d t^{2}}=0
$$

Multiplying (1.8) with $\Omega_{l}^{q}$, we see that the relations

$$
d x^{l}=a^{q} \Omega_{q}^{l} d t
$$

hold along the II-geodesic curves.

The $\left(\xi^{l}\right)$ may be interpreted as generalized parallel coordinates referred to II-geodesic coordinate axes. We will call $\left(\xi^{l}\right)$ the IIgeodesic parallel coordinates corresponding to $\omega_{m}^{l}(x)$.

2. Extension of the affine transformation group. From (1.8) and (1.9), we have

$$
d \xi^{l}=a_{m}^{2}(x) d x^{m}
$$

where

$$
a_{m}^{l}(x) \stackrel{\text { def }}{=} \omega_{m}^{l}(x) .
$$

From (2.1), we obtain

$$
\xi^{l}=a_{m}^{l}(x) x^{m}+a_{0}^{l}(x),
$$

where

$$
a_{0}^{l}(x) \stackrel{\text { def }}{=}-\int x^{m} d a_{m}^{l}(x)
$$


by integration by parts.

In order that (2.3) may be considered as an extended affine transformation, straight lines

$$
\frac{d^{2} x^{p}}{d t^{2}}=0
$$

must be transformed into II-geodesic curves corresponding to $\left\{a_{m}^{l}(x)\right\}$ :

$$
\frac{d^{2} \xi^{l}}{d t^{2}}=0
$$

Now, from (2.1), we have

$$
\frac{d \xi^{l}}{d t}=a_{m}^{l}(x) \frac{d x^{m}}{d t}, \quad \frac{d^{2} \xi^{l}}{d t^{2}}=\frac{d a_{m}^{l}(x)}{d t} \frac{d x^{m}}{d t}+a_{m}^{l}(x) \frac{d^{2} x^{m}}{d t^{2}} .
$$

Hence, by the demands (2.5) and (2.6), we must have

$$
0=d a_{m}^{l}(x) d x^{m_{c}}=d a_{m}^{l}(x) \wedge d x^{m}=\boldsymbol{d} \omega^{l}
$$

along the II-geodesic curves, where $\boldsymbol{d}$ is the exterior differential operator. (2.7) arises from the so-called equations of structure.

(2.5) and (2.6) and thus (2.7) demand us that $a_{0}^{l}(x)$ must be constant. For,

$$
\begin{aligned}
a_{0}^{l}(x) & =-\int x^{m} d a_{m}^{l}(x)=-\int d a_{m}^{l}(x) \int d x^{m}=-\iint\left\{d a_{m}^{l}(x) d x^{m}\right\} \\
& =-\iint\left\{-\frac{\partial a_{s}^{l}(x)}{\partial x^{r}} \frac{d x^{r}}{d t} \wedge \frac{d x^{m}}{d t}\right\} d t^{2}=-\iint\{0\} d t^{2}=0
\end{aligned}
$$

along the II-geodesic coordinate axes corresponding to $\left\{a_{m}^{l}(x)\right\}$. Thereby the condition for that the repeated integral may be converted into a double integral is satisfied and we may put

$$
d a_{m}^{l}(x) d x^{m}=d a_{m}^{l}(x) \wedge d x^{m},
$$

since their zero-values only are retained.

Thus we have finally

$$
\xi^{l}=a_{m}^{l}(x) x^{m}+a_{0}^{l}, \quad\left(a_{0}^{l}=\text { const. }\right)
$$

acompanied by the condition

$$
\left|a_{m}^{l}(x)\right| \neq 0 \text { in } A^{n}
$$

to which we will refer as extended affine transformation.

The totality of the extended affine transformations forms a group (5), which we will call the extended affine (transformation) group. The (5) contains the ordinary affine group (5) as subgroup.

The totality of the elements of $(5)$, which are free from $\mathbb{E}$, together with the unit transformation, forms a subgroup $\mathfrak{S}$ of $\mathscr{S}$, so that we have

$$
\mathscr{G}=\mathfrak{C S}=\mathfrak{S}=\mathfrak{S} \text {. }
$$

The geometry under (S) will be called the extended affine geometry.

\section{Expressions for $\xi^{l}$ in terms of $\boldsymbol{x}^{p}$}

The transformation formula (2.8) itself expresses $\left(\xi^{l}\right)$ in terms of $\left(x^{p}\right)$. Owing to (2.9), it admits of its inverse transformation (cf. (5.1)). 
II. Transformation of (0.1) into (0.2)

4. Transformation of $g^{i j} \frac{\partial}{\partial x^{i}} \frac{\partial}{\partial x^{j}}$ into $\frac{\partial^{2}}{\partial \xi^{l} \partial \xi^{l}}$. Being suggested by the known relations

$$
d s^{2}=g_{i j} d x^{i} d x^{j}=\omega^{l} \omega^{l}, \quad g_{i j}=\omega_{i}^{l} \omega_{j}^{l},
$$

we proceed as follows. We can readily prove the identity:

$$
g^{i j}(x)=\Omega_{l}^{i} \Omega_{l}^{j}
$$

which suggests:

Now it is known that

$$
g^{i j}(x) \frac{\partial}{\partial x^{i}} \frac{\partial}{\partial x^{j}}=\Omega_{l}^{i} \Omega_{l}^{j} \frac{\partial}{\partial x^{i}} \frac{\partial}{\partial x^{j}} .
$$

$$
\Omega_{l}^{i} \frac{\partial}{\partial x^{i}}=\frac{\partial}{\omega^{l}}
$$

where $\frac{\partial}{\omega^{2}}$ is a generalization of the partial derivative operator.

Owing to (1.10), we may rewrite (4.3) as follows:

$$
\Omega_{l}^{i} \frac{\partial}{\partial x^{i}}=\frac{\partial}{\partial \xi^{l}}
$$

adopting the II-geodesic parallel coordinates $\xi^{i}$.

Hence we have

$$
g^{i j}(x) \frac{\partial^{2}}{\partial x^{i} \partial x^{j}}=\frac{\partial^{2}}{\partial \xi^{l} \partial \xi^{l}} .
$$

\section{To transform $(0.1)$ into $(0.2)$}

The inverse transformation of (2.8) is

$$
x^{l}=\Omega_{p}^{l} \xi^{p}+x_{0}^{l}
$$

where

$$
x_{0}^{l}=-\Omega_{p}^{l} a_{0}^{p} .
$$

Introducing (4.5) and (5.1) into (0.1):

$$
g^{i j}(x) \frac{\partial^{2} u}{\partial x^{i} \partial x^{j}}+b^{l}(x) \frac{\partial u}{\partial x^{l}}+c(x) u=f(x),
$$

we obtain

$$
\varepsilon_{l} \frac{\partial^{2} u}{\partial \xi^{l} \partial \xi^{l}}+\bar{b}^{l}(\xi) \frac{\partial u}{\partial \xi^{l}}+\bar{c}(\xi) u=\bar{f}(\xi) .
$$

So far we have $\varepsilon_{l}=1$. But, if we confine ourselves to real $\xi^{l}$ only, some of $\varepsilon_{l}$ may be +1 and others -1 .

Thus we are led to find $\Omega_{\imath}^{i}$ from $g^{i j}$ except under extended affine transformations by a process similar to that by which we find $\omega_{m}^{l}$ from $g_{i j}$, assuming that

$$
\left|g^{i j}(x)\right| \neq 0, \quad g^{i j}(x) \in C^{\nu}
$$

\section{References}

[1] R. Courant und D. Hilbert: Methoden der mathematischen Physik. II, 223-288, 385-410 (1937). 
[2] T. Takasu: Erweiterung des Erlanger Programms durch Transformationsgruppenerweiterungen, Proc. Japan Acad., 34, 471-476 (1958).

[3] T. Takasu: Extended Euclidean geometry and extended equiform geometry under the extensions of respective transformation groups, I, Yokohama Math. J., 6, 1-88 (1958); II, ibid., 7, 1-32 (1959).

[4] T. Takasu: Extended affine geometry, Yokohama Math. J., 7 (1959) (under preparation).

[5] M. Uchiyama: A theory of differential equations viewed through group parameter extensions, I. A global theory of a system of total differential equations, Yokohama Math. J., 7, 43-56 (1959).

[6] M. Hukuhara and K. Nakamori: Modern Theory of Differential Equations, Part II, Kyoritsu-Shuppan \& Co., Tokyo (1959).

[7] S. S. Chern: Differentiable manifolds, Lecture note in Chicago University (1953).

[8] C. Ehresmann: Les Connexions Infinitésimales dans un Espace Fibré Différentiable, Colloque de Topologie, Bruxelles (Espace Fibrés), 29-55 (1950). 